Article

\title{
Time-Space Fractional Coupled Generalized Zakharov-Kuznetsov Equations Set for Rossby Solitary Waves in Two-Layer Fluids
}

\author{
Lei Fu ${ }^{1}$, Yaodeng Chen ${ }^{2}$ and Hongwei Yang ${ }^{1,2, *(D)}$ \\ 1 College of Mathematics and Systems Science, Shandong University of Science and Technology, \\ Qingdao 266590, China; fulei@sdust.edu.cn \\ 2 Key Laboratory of Meteorological Disaster (KLME), Ministry of Education and Collaborative Innovation \\ Center on Forecast and Evaluation of Meteorological Disasters (CIC-FEMD), Nanjing University of \\ Information Science and Technology, Nanjing 210044, China; keyu@nuist.edu.cn \\ * Correspondence: hwyang1979@163.com
}

Received: 7 December 2018; Accepted: 29 December 2018; Published: 3 January 2019

\begin{abstract}
In this paper, the theoretical model of Rossby waves in two-layer fluids is studied. A single quasi-geostrophic vortex equation is used to derive various models of Rossby waves in a one-layer fluid in previous research. In order to explore the propagation and interaction of Rossby waves in two-layer fluids, from the classical quasi-geodesic vortex equations, by employing the multi-scale analysis and turbulence method, we derived a new $(2+1)$-dimensional coupled equations set, namely the generalized Zakharov-Kuznetsov(gZK) equations set. The gZK equations set is an extension of a single ZK equation; they can describe two kinds of weakly nonlinear waves interaction by multiple coupling terms. Then, for the first time, based on the semi-inverse method and the variational method, a new fractional-order model which is the time-space fractional coupled gZK equations set is derived successfully, which is greatly different from the single fractional equation. Finally, group solutions of the time-space fractional coupled gZK equations set are obtained with the help of the improved $\left(G^{\prime} / G\right)$-expansion method.
\end{abstract}

Keywords: time-space fractional coupled generalized Zakharov-Kuznetsov equations set; Rossby waves; quasi-geostrophic vortex equations; two-layer fluids

\section{Introduction}

Although the existence of solitary waves has been known in hydrokinetics for about a century, it was not until recently that the theory was applied to wave phenomena in the atmospheric, ocean [1-6] and large lake dynamics system, such as solitary waves [7], internal gravity waves [8], internal Kelvin waves [9] and so on. Since Long(1964) derived the Korteweg de-Vries(KdV) equation on the positive pressure system, the isolated Rossby wave theory has been gradually developed [10-12], but in many complex atmospheric and oceanic systems, waves interact with each other. The problem of wave-wave interaction is a very important one in the earth fluid, and further study can deepen people's understanding of large-scale motion phenomena in the atmosphere, ocean and large lakes. There are many motion equations describing Rossby waves in a layer of fluid, such as one-dimensional $\mathrm{KdV}$ equation [13], MKdV equation [14], Boussinesq equation [15] and high-dimensional ZK equation [16], mZK equation [17], KP equation [18] and so on. The actual atmospheric ocean system is very complex, compared with the single equation of low dimension, the coupled equations set [19-21] of high dimension are more practical. Therefore, this paper derives the (2+1)-dimensional coupled generalized $\mathrm{ZK}(\mathrm{gZK})$ equations set from the classical quasi-geostrophic vorticity equations [22,23]. The gZK equation is a class of important high-dimensional nonlinear evolution equation in mathematical 
physics, the gZK equations set as the extension of a single equation can be used to describe the interaction of nonlinear Rossby waves in two-layer fluids [24-26].

Fractional calculus have been used to model atmospheric and oceanic systems, and these models have been found to be suitable to be described by fractional differential equations [27-29]. Standard mathematical models of integer derivatives, including nonlinear models, do not work well in many cases. For fractional-order equations set, most researchers only choose the time fractional-order equations set $[30,31]$, but few study the time-space fractional-order equations set, especially the time-space coupled fractional-order equations set. So, for the first time, we transform integral order coupled gZK equations set into fractional order coupled gZK equations set of space-time, and find that it has great research value.

Given the exact solution of nonlinear equations set [32,33] plays an important role in explaining some complex phenomena in physics, dynamics and other scientific fields, mathematicians and physicists have done a lot of researches on solving nonlinear equations set [34-41]. So far, researchers have proposed many effective solutions, such as the $\left(G^{\prime} / G\right)$-expansion method [42], the exp-function method [43], the Kudryashov method [21], the sun-equation method [44], the functional variable method [45], the modified extended Tanh method [46], Khater method [47], Godunov-type method [48], Lie group analysis method $[49,50]$ and so on. We choose the modified $\left(G^{\prime} / G\right)$-expansion method to solve the time-space fractional coupled gZK equations set, and get several different kinds of solutions.

\section{Derivation of Coupled gZK Equations Set}

A single set of quasi-geostrophic vortex equation is usually used to derive various equations which can be used to research Rossby solitary waves [51] in a single layer of the atmosphere and ocean. For the waves in two-layer fluids, they are divided into upper and lower layers as shown in Figure 1, but the isolated waves between the two layers do not propagate separately but interact with each other, this is where the coupled equations set model comes in.

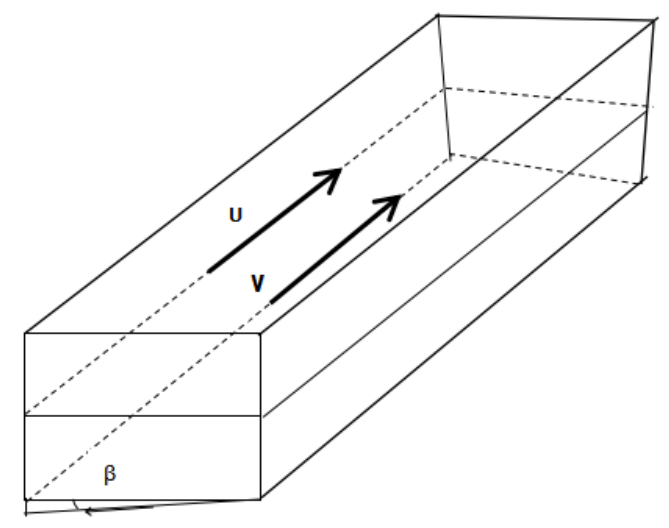

Figure 1. Model geometry for the two-layer fluids, $\mathrm{U}$ and $\mathrm{V}$ are the basic flows of the upper and lower layers, $\beta$ represents the Coriolis parameter.

In the first place, for the exploration of the Rossby waves propagation and action between two-layer fluids, the coupled gZK equations set of the objective function is derived from the following two layers of quasi-geostrophic vortex equations set by using the multi-scale analysis and turbulence method

$$
\begin{aligned}
& q_{A t}+J\left(\psi_{A}, q_{A}\right)+\beta \psi_{A x}=0, \\
& q_{B t}+J\left(\psi_{B}, q_{B}\right)+\beta \psi_{B x}=0,
\end{aligned}
$$


where $\psi_{A}, \psi_{B}$ are the stream functions of the upper fluid and the lower fluid respectively, $J[a, b]$ is the Jacobi operator and $J(a, b)=a_{x} b_{y}-b_{x} a_{y}$ and $\beta$ represents the Coriolis parameter, as well as

$$
\begin{aligned}
& q_{A}=\psi_{A x x}+\psi_{A y y}+F\left(\psi_{B}-\psi_{A}\right), \\
& q_{B}=\psi_{B x x}+\psi_{B y y}+F\left(\psi_{A}-\psi_{B}\right) .
\end{aligned}
$$

Then the Equation (1) colorredcan be expanded to the following form

$$
\begin{aligned}
& \psi_{A x x t}+\psi_{A y y t}+F\left(\psi_{B}-\psi_{A}\right)_{t}+\psi_{A x}\left[\psi_{A x x y}+\psi_{A y y y}+F\left(\psi_{B}-\psi_{A}\right)_{y}\right] \\
& -\left[\psi_{A x x x}+\psi_{A y y x}+F\left(\psi_{B}-\psi_{A}\right)_{x}\right] \psi_{A y}+\beta \psi_{A x}=0, \\
& \psi_{B x x t}+\psi_{B y y t}+F\left(\psi_{A}-\psi_{B}\right)_{t}+\psi_{B x}\left[\psi_{B x x y}+\psi_{B y y y}+F\left(\psi_{A}-\psi_{B}\right)_{y}\right] \\
& -\left[\psi_{B x x x}+\psi_{B y y x}+F\left(\psi_{A}-\psi_{B}\right)_{x}\right] \psi_{B y}+\beta \psi_{B x}=0,
\end{aligned}
$$

with $F$ represents the weak coupling coefficient between two-layer fluids [22].

In the next place, so as to derive the gZK type equations set, we take the long wave approximation in the $x$ direction, then the stream functions $\psi_{A}$ and $\psi_{B}$ can be divided into the basic stream functions and the disturbance stream functions, written as

$$
\begin{aligned}
& \psi_{A}=\phi_{A_{0}}(y)+\phi_{A}(x, y, t)=\left(U_{A_{0}}+c_{0} y\right)+\phi_{A}(x, y, t), \\
& \psi_{B}=\phi_{B_{0}}(y)+\phi_{B}(x, y, t)=\left(U_{B_{0}}+c_{0} y\right)+\phi_{B}(x, y, t) .
\end{aligned}
$$

Suppose the coupling between the two-layer fluids is weak, and the rotation effect of the Earth is very small, therefore we can adopt the following space-time transformation

$$
\begin{gathered}
F=\varepsilon F_{0}, \quad \beta=\varepsilon^{2} \beta, \\
X=\varepsilon\left(x-c_{0} t\right), \quad Y=\varepsilon y, \quad T=\varepsilon^{3} t,
\end{gathered}
$$

where $\varepsilon$ is a small parameter.

Under the premise of Equations (5) and (6), Equation (3) can be rewritten as the following form

$$
\begin{aligned}
& -c_{0} \varepsilon^{3} \phi_{A X X X}+\varepsilon^{5} \phi_{A X X T}-c_{0} \varepsilon^{3} \phi_{A Y Y X}-2 c_{0} \varepsilon^{2} \phi_{A Y y X}-c_{0} \varepsilon \phi_{A y y}+\varepsilon^{5} \phi_{A Y Y T}+2 \varepsilon^{4} \phi_{A Y y T} \\
& +\varepsilon^{3} \phi_{A y y T}+\varepsilon^{4} F_{0}\left(\phi_{B T}-\phi_{A T}\right)-c_{0} \varepsilon^{2} F_{0}\left(\phi_{B X}-\phi_{A X}\right)+\varepsilon \phi_{A X}\left[\varepsilon^{3} \phi_{A X X Y}+\varepsilon^{2} \phi_{A X X y}+\right. \\
& \phi_{A_{0} y y y}+\varepsilon^{3} \phi_{A Y Y Y}+3 \varepsilon^{2} \phi_{A Y Y y}+3 \varepsilon \phi_{A Y y y}+\phi_{A y y y}+\varepsilon F_{0}\left(\phi_{B_{0} y}-\phi_{A_{0} y}\right)+\varepsilon^{2} F_{0}\left(\phi_{B Y}-\phi_{A y}\right) \\
& \left.+\varepsilon F_{0}\left(\phi_{B y}-\phi_{A y}\right)\right]-\left[\varepsilon^{3} \phi_{A X X X}+\varepsilon^{3} \phi_{A Y Y X}+2 \varepsilon^{2} \phi_{A Y y X}+\varepsilon^{2} F_{0}\left(\phi_{B X}-\phi_{A X}\right)\right]\left(\phi_{A_{0} y}\right. \\
& \left.+\varepsilon \phi_{A Y}+\phi_{A y}\right)+\varepsilon^{4} \beta_{1} \phi_{A X}=0 \\
& -c_{0} \varepsilon^{3} \phi_{B X X X}+\varepsilon^{5} \phi_{B X X T}-c_{0} \varepsilon^{3} \phi_{A Y Y X}-2 c_{0} \varepsilon^{2} \phi_{B Y y X}-c_{0} \varepsilon \phi_{B y y X}+\varepsilon^{5} \phi_{B Y Y T}+2 \varepsilon^{4} \phi_{B Y y T} \\
& +\varepsilon^{3} \phi_{B y y T}+\varepsilon^{4} F_{0}\left(\phi_{A T}-\phi_{B T}\right)-c_{0} \varepsilon^{2} F_{0}\left(\phi_{A X}-\phi_{B X}\right)+\varepsilon \phi_{B X}\left[\varepsilon^{3} \phi_{B X X Y}+\varepsilon^{2} \phi_{B X X y}+\right. \\
& \phi_{B_{0} y y y}+\varepsilon^{3} \phi_{B Y Y Y}+3 \varepsilon^{2} \phi_{B Y Y y}+3 \varepsilon \phi_{B Y y y}+\phi_{B y y y}+\varepsilon F_{0}\left(\phi_{A_{0} y}-\phi_{B_{0} y}\right)+\varepsilon^{2} F_{0}\left(\phi_{A Y}-\phi_{B y}\right) \\
& \left.+\varepsilon F_{0}\left(\phi_{A y}-\phi_{B y}\right)\right]-\left[\varepsilon^{3} \phi_{B X X X}+\varepsilon^{3} \phi_{B Y Y X}+2 \varepsilon^{2} \phi_{B Y y X}+\varepsilon^{2} F_{0}\left(\phi_{A X}-\phi_{B X}\right)\right]\left(\phi_{B_{0} y}\right. \\
& \left.+\varepsilon \phi_{B Y}+\phi_{B y}\right)+\varepsilon^{4} \beta_{1} \phi_{B X}=0,
\end{aligned}
$$

where the perturbation stream functions have the following series expansion forms

$$
\begin{aligned}
& \phi_{A}=\varepsilon \phi_{A_{1}}(X, Y, T)+\varepsilon^{2} \phi_{A_{2}}(X, Y, T)+\varepsilon^{3} \phi_{A_{3}}(X, Y, T)+o\left(\varepsilon^{4}\right), \\
& \phi_{B}=\varepsilon \phi_{B_{1}}(X, Y, T)+\varepsilon^{2} \phi_{B_{2}}(X, Y, T)+\varepsilon^{3} \phi_{B_{3}}(X, Y, T)+o\left(\varepsilon^{4}\right) .
\end{aligned}
$$

By substituting Equation (8) into Equation (7), we obtained the following equations about small parameter $\varepsilon$ 


$$
\begin{aligned}
& \varepsilon^{2}:\left\{\begin{array}{l}
-\phi_{A_{0 y y y}} \phi_{A_{1 X}}-c_{0} \phi_{A_{1 y y X}}+\phi_{A_{0 y}} \phi_{A_{1 y y X}}=0, \\
-\phi_{B_{0 y y y}} \phi_{B_{1 X}}-c_{0} \phi_{B_{1 y y X}}+\phi_{B_{0 y}} \phi_{B_{1 y y X}}=0,
\end{array}\right. \\
& \varepsilon^{3}:\left\{\begin{array}{l}
-\phi_{A_{0 y y y}} \phi_{A_{2 X}}-c_{0} \phi_{A_{2 y y X}}+\phi_{A_{0 y}} \phi_{A_{2 y y X}}-2 \phi_{A_{1 Y y X}}\left(\phi_{A_{0 y}}-c_{0}\right) \\
+\phi_{A_{1 X}} \phi_{A_{1 y y y}}-\phi_{A_{1 y y X}} \phi_{A_{1 y}}+F_{0} \phi_{B_{1 X}}\left(\phi_{A_{0 y}}-c_{0}\right)+F_{0} \phi_{A_{1 X}}\left(\phi_{B_{0 y}}-c_{0}\right)=0, \\
-\phi_{B_{0 y y y}} \phi_{B_{2 X}}-c_{0} \phi_{B_{2 y y X}}+\phi_{B_{0 y}} \phi_{B_{2 y y X}}-2 \phi_{B_{1 Y y X}}\left(\phi_{B_{0 y}}-c_{0}\right) \\
+\phi_{B_{1 X}} \phi_{B_{1 y y y}}-\phi_{B_{1 y y X}} \phi_{B_{1 y}}+F_{0} \phi_{A_{1 X}}\left(\phi_{B_{0 y}}-c_{0}\right)+F_{0} \phi_{B_{1 X}}\left(\phi_{A_{0 y}}-c_{0}\right)=0,
\end{array}\right.
\end{aligned}
$$

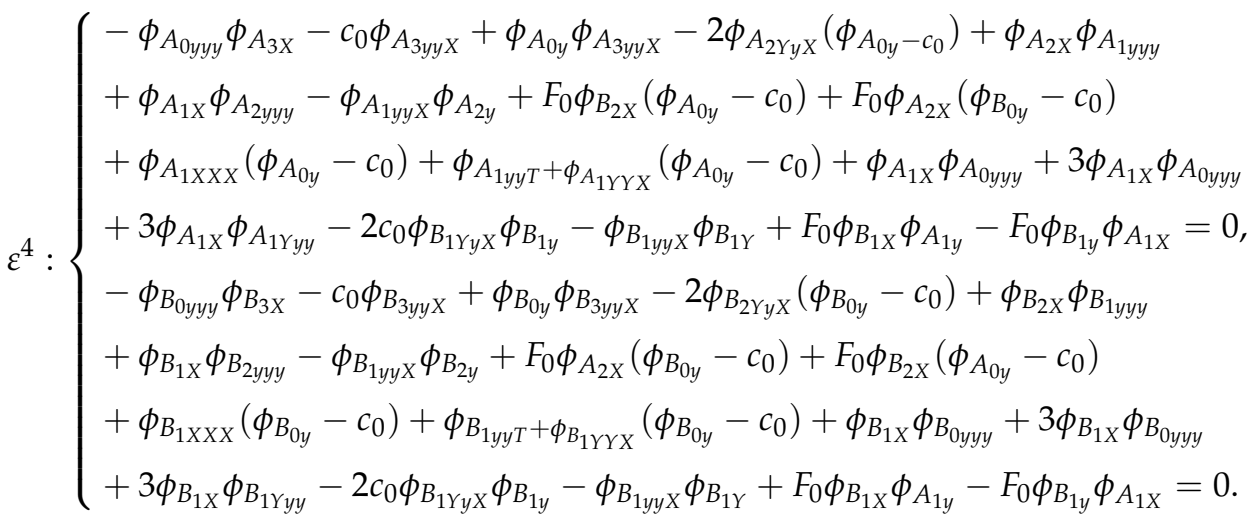

In Equation (9), it is easy to find that $\phi_{A_{1}}$ and $\phi_{A_{2}}$ have the following separable variables form solutions

$$
\begin{aligned}
& \phi_{A_{1}}=A_{1}(X, Y, T) B_{1}(y) \equiv A_{1} B_{1}, \\
& \phi_{B_{1}}=A_{2}(X, Y, T) B_{2}(y) \equiv A_{2} B_{2} .
\end{aligned}
$$

Substituting (12) into Equation (9), we get the following equations about variable $y$

$$
\begin{aligned}
& U_{A_{0 y}} B_{1 y}-U_{A_{0 y y}} B_{1}+C_{1}=0, \\
& U_{B_{0 y}} B_{2 y}-U_{B_{0 y y}} B_{2}+C_{2}=0,
\end{aligned}
$$

where $C_{1}, C_{2}$ are arbitrary constants. Applying Equations (12) and (13) to Equation (10), integrating with respect to $X$ once, cancel out the integral function, and we get the following equations

$$
\begin{aligned}
& 2 U_{A_{0 y}}\left(B_{1} \partial_{y y}-B_{1}\right) \phi_{A_{2}}+B_{1}\left[A_{1}^{2}\left(B_{1} B_{1 y y y}-B_{1 y} B_{1 y y}\right)\right. \\
& \left.-4 U_{A_{0 y}} A_{1 Y} B_{1 y}-2 F_{0}\left(U_{B_{0 y}} B_{1} A_{1}-U_{A_{0 y}} B_{2} A_{2}\right)\right]=0 \\
& 2 U_{B_{0 y}}\left(B_{2} \partial_{y y}-B_{2}\right) \phi_{B_{2}}+B_{2}\left[A_{2}^{2}\left(B_{2} B_{2 y y y}-B_{2 y} B_{2 y y}\right)\right. \\
& \left.-4 U_{B_{0 y}} A_{2 Y} B_{2 y}-2 F_{0}\left(U_{A_{0 y}} B_{2} A_{2}-U_{B_{0 y}} B_{1} A_{1}\right)\right]=0 .
\end{aligned}
$$

It's easy to see from this set of equations that

$$
\begin{aligned}
& \phi_{A_{2}}=\left(a_{1} A_{1}^{2}+a_{2} A_{1 Y}+a_{3} A_{1}+a_{4} A_{2}\right) B_{1}, \\
& \phi_{B_{2}}=\left(b_{1} A_{2}^{2}+b_{2} A_{2 Y}+b_{3} A_{2}+b_{4} A_{1}\right) B_{2}
\end{aligned}
$$

where $a_{i}, b_{i}, i=1,2,3,4$ are functions of $y$

$$
\begin{array}{ll}
a_{1}=\frac{B_{1} B_{1 y y y}-B_{1 y} B_{1 y y}}{4 U_{A_{0 y}} B_{1 y y}}, \quad a_{2}=-\frac{B_{1 y}}{B_{1 y y}}, \quad a_{3}=-\frac{F_{0} U_{B_{0 y}}}{2 U_{A_{0 y}} B_{1 y y}}, \quad a_{4}=\frac{F_{0} B_{2}}{2 B_{1 y y}}, \\
b_{1}=\frac{B_{2} B_{2 y y y}-B_{2 y} B_{2 y y}}{4 U_{B_{0 y}} B_{2 y y}}, \quad a_{2}=-\frac{B_{2 y}}{B_{2 y y}}, \quad b_{3}=-\frac{F_{0} U_{A_{0 y}}}{2 U_{B_{0 y}} B_{2 y y}}, \quad b_{4}=\frac{F_{0} B_{1}}{2 B_{2 y y}} .
\end{array}
$$


In the end, after substituting Equation (12), Equation (15) and $\psi_{A_{3}}=\psi_{B_{3}}=0$ into Equation (9), the integrating from 0 to $y_{0}$ of the resulting, the following coupled gZK equations set can be obtained though simple calculation.

$$
\begin{aligned}
& A_{1 T}+c_{1}\left(A_{1} A_{2}\right)_{X}+c_{2}\left(A_{1}^{2}\right)_{X}+c_{3}\left(A_{2}^{2}\right)_{X}+c_{4} A_{1 X Y}+c_{5} A_{2 X Y}+c_{6}\left(A_{1}^{2}\right)_{X Y}+c_{7} A_{1 X X X}+c_{8} A_{1 X Y Y}=0, \\
& A_{2 T}+d_{1}\left(A_{1} A_{2}\right)_{X}+d_{2}\left(A_{1}^{2}\right)_{X}+d_{3}\left(A_{2}^{2}\right)_{X}+d_{4} A_{1 X Y}+d_{5} A_{2 X Y}+d_{6}\left(A_{2}^{2}\right)_{X Y}+d_{7} A_{2 X X X}+d_{8} A_{2 X Y}=0,
\end{aligned}
$$

where

$$
\begin{aligned}
& c_{1}=\int_{0}^{y_{0}}\left(\frac{a_{4} B_{1} B_{1 y y y}+F_{0} B_{1} B_{1 y}}{B_{1 y y}}-a_{4} B_{1}\right) d y, \quad c_{2}=\int_{0}^{y_{0}}\left(\frac{F_{0} U_{B_{0 y}}}{B_{1 y y}}-2 a_{3} B_{1}-2 B_{1 y}\right) d y, \\
& c_{3}=2 \int_{0}^{y_{0}} \frac{b_{1} F_{0} U_{A_{0 y}} B_{2}}{B_{1 y y}} d y, c_{4}=\int_{0}^{y_{0}} \frac{a_{3} B_{1 y}+a_{2} F_{0} U_{B_{0 y}} B_{1}}{B_{1 y y}} d y, c_{5}=\int_{0}^{y_{0}} \frac{a_{4} B_{1 y}+b_{2} F_{0} U_{A_{0 y}} B_{2}}{B_{1 y y}} d y, \\
& c_{6}=\int_{0}^{y_{0}}\left(\frac{a_{2} B_{1} B_{1 y y}-2 B_{1 y}^{2}+2 a_{1} B_{1 y}}{B_{1 y}}-a_{2} B_{1}\right) d y, c_{7}=\int_{0}^{y_{0}} \frac{U_{A_{0 y}} B_{1}}{B_{1 y y}} d y, c_{8}=\int_{0}^{y_{0}} \frac{a_{2} B_{1 y}+U_{A_{0 y}} B_{1}}{B_{1 y y}}, \\
& d_{1}=\int_{0}^{y_{0}}\left(\frac{b_{4} B_{2} B_{2 y y}+F_{0} B_{2} B_{2 y}}{B_{2 y y}}-b_{4} B_{2}\right) d y, \quad d_{3}=\int_{0}^{y_{0}}\left(\frac{b_{1} F_{0} U_{A_{0 y} B_{2}}}{B_{2 y y}}-2 a_{3} B_{2}-2 a_{3} B_{2 y}\right) d y, \\
& d_{2}=2 \int_{0}^{y_{0}} \frac{a_{1} F_{0} U_{B_{0 y}} B_{1}}{B_{2 y y}}, d_{4}=\int_{0}^{y_{0}} \frac{b_{4} B_{2 y}+a_{2} F_{0} U_{B_{0 y}} B_{1}}{B_{2 y y}} d y, d_{5}=\int_{0}^{y_{0}} \frac{b_{3} B_{2 y}+b_{2} F_{0} U_{B_{0 y}} B_{2}}{B_{2 y y}} d y, \\
& d_{6}=\int_{0}^{y_{0}}\left(\frac{b_{2} B_{2} B_{2 y y y}-2 B_{2 y}^{2}+2 b_{1} B_{2 y}}{B_{2 y y}}-b_{2} B_{2} B_{2 y y}\right) d y, d_{7}=\int_{0}^{y_{0}} \frac{U_{B_{0} y} B_{2}}{B_{2 y y}} d y, d_{8}=\int_{0}^{y_{0}} \frac{b_{2} B_{2 y}+U_{B_{0} y} B_{2}}{B_{2 y y}} .
\end{aligned}
$$

Remark 1. The coupled gZK equations set is the extension of a single ZK equation and a class of important nonlinear evolution equations of high dimension. They describe two kinds of weakly nonlinear waves interaction with each other and the interaction between two waves is reflected in multiple coupling terms $\left(A_{1} A_{2}\right)_{X},\left(A_{1}^{2}\right)_{X Y}$ and $\left(A_{2}^{2}\right)_{X Y}$.

\section{The Time-Space Fractional Coupled gZK Equations set}

In previous work, we only derived a single fractional-order equation, but here we will apply the semi-inverse method and the variational method [52-54] to derive the coupled fractional-order equations set for the first time, and obtain a new fractional-order coupled equations set, namely time-space fractional coupled gZK equations set. colorredFor the ease of understanding, some definitions and properties of fractional order are introduced before demonstrating the specific derivation process.

Definition 1 ([52]). Modified Rieman-Liouville derivative

$$
D_{t}^{\alpha} f(t)=\left\{\begin{array}{l}
\frac{1}{\Gamma(1-\alpha)} \frac{d}{d t} \int_{0}^{t}(t-\delta)^{-\alpha}(f(\delta)-f(0)) d \delta, \quad 0<\alpha<1, \\
\left(f^{(n)(x)}\right)^{(\alpha-n)}, \quad n \leq \alpha<n+1, n \geq 1,
\end{array}\right.
$$

where $f(t)$ is a continuous function.

Definition 2 ([42]). Assume that $f(t)$ denotes a continuous $R \rightarrow R$ function, we use the following equality for the integral

$$
D_{t}^{\alpha} f(t)=\frac{1}{\Gamma(\alpha)} \int_{0}^{t}(t-\zeta)^{-1} f(\zeta) d \zeta=\frac{1}{\Gamma(1+\alpha)} \frac{d}{d t} \int_{0}^{t} f(\zeta)(d \zeta)^{\alpha}, \quad 0<\alpha \leq 1
$$

Property 1 ([54]). Integral property of fractional order equation

$$
\int_{a}^{t}(d \tau)^{\alpha} f(\tau)=\alpha \int_{a}^{t} d \tau(t-\tau)^{\alpha} f(\tau)
$$


Property 2 ([54]). Integration by parts property of fractional order equation

$$
\begin{gathered}
\int_{a}^{b}(d \tau)^{\alpha} f(t) D_{t}^{\alpha} g(t)=\frac{1}{\Gamma(1-\alpha)}\left[\left.g(t) f(t)\right|_{a} ^{b}-\int_{a}^{b}(d \tau)^{\alpha} g(t) D_{t}^{\alpha} f(t)\right], \\
f(t), g(t) \in[a, b] .
\end{gathered}
$$

Introducing two potential functions $U(X, Y, T)$ and $V(X, Y, T)$, and their relationship to $A_{1}$ and $A_{2}$ is that $A_{1}=U_{X}, A_{2}=V_{X}$, substituting these two expressions into Equation (17) separately, the potential equations of the coupled gZK equations set have the form as

$$
\begin{aligned}
& U_{X T}+c_{1}\left(v_{X} V\right)_{X}+c_{2}\left(U_{X}^{2}\right)_{X}+c_{3}\left(V_{X}^{2}\right)_{X}+c_{4} U_{X X Y} \\
& +c_{5} V_{X X Y}+c_{6}\left(U_{X}^{2}\right)_{X Y}+c_{7} U_{X X X X}+c_{8} U_{X X Y Y}=0, \\
& V_{X T}+d_{1}\left(V_{X} U\right)_{X}+d_{2}\left(U_{X}^{2}\right)_{X}+d_{3}\left(V_{X}^{2}\right)_{X}+d_{4} U_{X X Y} \\
& +d_{5} V_{X X Y}+d_{6}\left(V_{X}^{2}\right)_{X Y}+d_{7} V_{X X X X}+d_{8} V_{X X Y Y}=0 .
\end{aligned}
$$

Further, the semi-inverse method was applied to derive the Lagrangian equations of coupled gZK equations, functional of the Equation (19) could be written as

$$
\begin{aligned}
J(U, V)= & \int_{R} d X \int_{R} d Y \int_{T} d T\left\{U \left[m_{1} U_{X T}+m_{2} c_{1}\left(U_{X} V\right)_{X}+m_{3} c_{2} U_{X} U_{X X}+m_{4} c_{3} V_{X} V_{X X}\right.\right. \\
& \left.+m_{5} c_{4} U_{X X Y}+m_{6} c_{5} V_{X X Y}+m_{7} c_{6}\left(U_{X}^{2}\right)_{X Y}+m_{8} c_{7} U_{X X X X}+m_{9} c_{8} U_{X X Y Y}\right] \\
& +V\left[n_{1} V_{X T}+n_{2} d_{1}\left(U_{X} V\right)_{X}+n_{3} d_{2} U_{X} U_{X X}+n_{4} d_{3} V_{X} V_{X X}+n_{5} d_{4} U_{X X Y}\right. \\
& \left.\left.+n_{6} d_{5} V_{X X Y}+n_{7} d_{6}\left(V_{X}^{2}\right)_{X Y}+n_{8} d_{7} V_{X X X X}+n_{9} d_{8} V_{X X Y Y}\right]\right\}
\end{aligned}
$$

with $m_{i}, n_{i}, i=1, \ldots, 9$ are Lagrange coefficients and will be calculated later to determine the exact values. Carrying out the integration by parts in Equation (20) and taking $\left.U_{X}\right|_{R}=\left.U_{Y}\right|_{R}=\left.U_{T}\right|_{T}=$ $\left.V_{X}\right|_{R}=\left.V_{Y}\right|_{R}=\left.V_{T}\right|_{T}=0$, the functional be rewritten as

$$
\begin{aligned}
J(U, V)= & \int_{R} d X \int_{R} d Y \int_{T} d T\left\{\left[-m_{1} U_{X} U_{T}-m_{2} c_{1} U_{X}^{2} V_{X}-\frac{1}{2} m_{3} c_{2} U_{X}^{3}-\frac{1}{2} m_{4} c_{3} U_{X} V_{X}^{2}\right.\right. \\
& \left.-m_{5} c_{4} U_{X} U_{X Y}-m_{6} c_{5} U_{X} V_{X Y}-m_{7} c_{6}\left(U_{X}^{2}\right) U_{X Y}-m_{8} c_{7} U_{X X}^{2}-m_{9} c_{8} U_{X Y}^{2}\right] \\
& -\left[n_{1} V_{X} V_{T}+n_{2} d_{1} V_{X}^{2} U_{X}+\frac{1}{2} n_{3} d_{2} V_{X}^{3}-\frac{1}{2} n_{4} d_{3} V_{X} U_{X}^{2}+n_{5} d_{4} V_{X} V_{X Y}\right. \\
& \left.\left.+n_{6} d_{5} V_{X} U_{X Y}+n_{7} d_{6} V_{X}^{2} V_{X Y}+n_{8} d_{7} V_{X X}^{2}+n_{9} d_{8} V_{X Y}^{2}\right]\right\} .
\end{aligned}
$$

Using the variational method for this functional equation, integrating by parts to optimize this variational, the resulting forms are expressed as

$$
\begin{aligned}
& -2 m_{1} U_{X T}-2 m_{2} c_{1}\left(U_{X} V\right)_{X}-3 m_{3} c_{2}\left(U_{X}^{2}\right)_{X}-3 m_{4} c_{3}\left(V_{X}^{2}\right)_{X}-2 m_{5} c_{4} U_{X X Y} \\
& -m_{6} c_{5} V_{X X Y}-4 m_{7} c_{6}\left(U_{X}^{2}\right)_{X Y}+m_{8} c_{7} U_{X X X X}-m_{9} c_{8} U_{X X Y Y}=0, \\
& -2 n_{1} V_{X T}-2 n_{2} d_{1}\left(V_{X} U\right)_{X}-3 n_{3} d_{2}\left(U_{X}^{2}\right)_{X}-3 n_{4} d_{3}\left(V_{X}^{2}\right)_{X}-2 n_{5} d_{4} U_{X X Y} \\
& -n_{6} d_{5} V_{X X Y}-4 n_{7} d_{6}\left(V_{X}^{2}\right)_{X Y}+n_{8} d_{7} V_{X X X X}-n_{9} d_{8} V_{X X Y Y}=0 .
\end{aligned}
$$

Since the Equations (22) and (19) are equal, the values of all the Lagrangian constants in the equations can be obtained, $m_{1}=m_{2}=m_{5}=n_{1}=n_{2}=n_{5}=-\frac{1}{2}, m_{3}=m_{4}=n_{3}=n_{4}=-\frac{1}{3}, m_{7}=$ $n_{7}=-\frac{1}{4}, m_{8}=n_{8}=1, m_{6}=m_{9}=n_{6}=n_{9}=-1$. Thus, the Lagrangian forms $[55,56]$ of the integer order coupled gZK equations set is, 


$$
\begin{aligned}
I_{1} & =\frac{1}{2} U_{X} U_{T}+\frac{1}{2} c_{1} U_{X}^{2} V_{X}+\frac{1}{3} c_{2} U_{X}^{3}+\frac{1}{3} c_{3} U_{X}^{2} V_{X}+c_{4} U_{X} U_{X Y} \\
& +\frac{1}{2} c_{5} U_{X} V_{X Y}+\frac{1}{4} c_{6}\left(U_{X}^{2}\right) U_{X Y}-c_{7} U_{X X}^{2}+c_{8} U_{X Y}^{2}=0 \\
I_{2} & =\frac{1}{2} V_{X} V_{T}+\frac{1}{2} d_{1} V_{X}^{2} U_{X}+\frac{1}{3} d_{2} V_{X}^{3}+\frac{1}{3} d_{3} V_{X}^{2} U_{X}+d_{4} V_{X} V_{X Y} \\
& +\frac{1}{2} d_{5} V_{X} U_{X Y}+\frac{1}{4} d_{6}\left(V_{X}^{2}\right) V_{X Y}-d_{7} V_{X X}^{2}+d_{8} V_{X Y}^{2}=0 .
\end{aligned}
$$

The fractional variational problem of Lagrange was obtained [57]. A natural generalization of Agrawal's approach [53,58-60], was applied to the fractional calculus of constrained systems. In order to obtain time-space fractional gZK equation set, we use the Lagrangian to minimize certain functionals which will naturally contain fractional derivative terms. Analogously, based on the Definition 1 and Agrawal's method, the Lagrangian forms of the time-space fractional coupled gZK equations are given as

$$
\begin{aligned}
F_{1} & =\frac{1}{2} D_{T}^{\alpha} U \times D_{X}^{\beta} U+\frac{1}{2} c_{1}\left(D_{X}^{\beta} U\right)^{2} D_{X}^{\beta} V+\frac{1}{3} c_{2}\left(D_{X}^{\beta} U\right)^{3}+\frac{1}{3} c_{3}\left(D_{X}^{\beta} V\right)^{2} D_{X}^{\beta} U+c_{4} D_{X}^{\beta} U \times D_{X Y}^{\beta \omega} U \\
& +\frac{1}{2} c_{5} D_{X}^{\beta} U \times D_{X Y}^{\beta \omega} V+\frac{1}{4} c_{6}\left(D_{X}^{\beta} U\right)^{2} D_{X Y}^{\beta \omega} V-c_{7}\left(D_{X}^{2 \beta} U\right)^{2}+c_{8}\left(D_{X Y}^{\beta \omega} U\right)^{2}=0, \\
F_{2} & =\frac{1}{2} D_{T}^{\alpha} V \times D_{X}^{\beta} V+\frac{1}{2} d_{1}\left(D_{X}^{\beta} V\right)^{2} D_{X}^{\beta} U+\frac{1}{3} d_{2}\left(D_{X}^{\beta} V\right)^{3}+\frac{1}{3} d_{3}\left(D_{X}^{\beta} U\right)^{2} D_{X}^{\beta} V+d_{4} D_{X}^{\beta} V \times D_{X Y}^{\beta \omega} V \\
& +\frac{1}{2} d_{5} D_{X}^{\beta} V \times D_{X Y}^{\beta \omega} U+\frac{1}{4} d_{6}\left(D_{X}^{\beta} V\right)^{2} D_{X Y}^{\beta \omega} U-d_{7}\left(D_{X}^{2 \beta} V\right)^{2}+d_{8}\left(D_{X Y}^{\beta \omega} V\right)^{2}=0,
\end{aligned}
$$

where $D_{X Y}^{\beta \omega} f=D_{Y}^{\omega}\left[D_{X}^{\beta} f\right], D_{X}^{2 \beta} f=D_{X}^{\beta}\left[D_{X}^{\beta} f\right]$.

It's similar to what we did for the integral order equation, the functional of the time-space coupled gZK equations set has the form

$$
J_{F}(U, V)=\int_{R}(d X)^{\beta} \int_{R}(d Y)^{\omega} \int_{T}(d T)^{\alpha}\left(F_{1}+F_{2}\right) .
$$

and the variation of functional Equation (24) leads to

$$
\begin{aligned}
\delta J_{F}(U, V)= & \int_{R}(d X)^{\beta} \int_{R}(d Y)^{\omega} \int_{T}(d T)^{\alpha}\left[D_{T}^{\alpha}\left(\frac{\partial F_{1}}{\partial D_{T}^{\alpha} U}\right)+D_{X}^{\beta}\left(\frac{\partial F_{1}}{\partial D_{X}^{\beta} U}\right)+D_{Y}^{\omega}\left(\frac{\partial F_{1}}{\partial D_{X}^{\beta} U}\right)\right. \\
& \left.-D_{X}^{2 \beta}\left(\frac{\partial F_{1}}{\partial D_{X}^{2 \beta}} U\right)\right] \delta J U+\int_{R}(d X)^{\beta} \int_{R}(d Y)^{\omega} \int_{T}(d T)^{\alpha}\left[D_{T}^{\alpha}\left(\frac{\partial F_{2}}{\partial D_{T}^{\alpha} V}\right)\right. \\
& \left.+D_{X}^{\beta}\left(\frac{\partial F_{2}}{\partial D_{X}^{\beta} V}\right)+D_{Y}^{\omega}\left(\frac{\partial F_{2}}{\partial D_{X}^{\beta} V}\right)-D_{X}^{2 \beta}\left(\frac{\partial F_{2}}{\partial D_{X}^{2 \beta}} V\right)\right] \delta J V .
\end{aligned}
$$

According to the properties introduced at the beginning, integrating the Equation (26) by parts and making $\delta J_{F}(U, V)=0$, optimizing the variation of the function, the following form Euler-Lagrange equations [53] for the time-space fractional coupled gZK equations set can be given

$$
\begin{aligned}
& D_{T}^{\alpha}\left(\frac{\partial F_{1}}{\partial D_{T}^{\alpha} U}\right)+D_{X}^{\beta}\left(\frac{\partial F_{1}}{\partial D_{X}^{\beta} U}\right)+D_{Y}^{\omega}\left(\frac{\partial F_{1}}{\partial D_{X}^{\beta} U}\right)-D_{X}^{2 \beta}\left(\frac{\partial F_{1}}{\partial D_{X}^{2 \beta}} U\right)=0, \\
& D_{T}^{\alpha}\left(\frac{\partial F_{2}}{\partial D_{T}^{\alpha} V}\right)+D_{X}^{\beta}\left(\frac{\partial F_{2}}{\partial D_{X}^{\beta} V}\right)+D_{Y}^{\omega}\left(\frac{\partial F_{2}}{\partial D_{X}^{\beta} V}\right)-D_{X}^{2 \beta}\left(\frac{\partial F_{2}}{\partial D_{X}^{2 \beta}} V\right)=0 .
\end{aligned}
$$


The last step is to plug expressions for $F_{1}, F_{2}$ given by Equation (24) and fractional potential functions $D_{X}^{\beta} U(X, Y, T)=u(X, Y, T), D_{X}^{\beta} V(X, Y, T)=v(X, Y, T)$ in this equation, the final equations set is

$$
\begin{aligned}
& D_{T}^{\alpha} u+c_{1} D_{X}^{\beta}(u v)+c_{2} D_{X}^{\beta}\left(u^{2}\right)+c_{3} D_{X}^{\beta}\left(v^{2}\right)+c_{4} D_{X}^{\beta} D_{Y}^{\omega} u \\
& +c_{5} D_{X}^{\beta} D_{Y}^{\omega} v+c_{6} D_{X}^{\beta} D_{Y}^{\omega}\left(u^{2}\right)+c_{7} D_{X}^{3 \beta} u+c_{8} D_{X}^{\beta} D_{Y}^{2 \omega} v=0, \\
& D_{T}^{\alpha} v+d_{1} D_{X}^{\beta}(u v)+d_{2} D_{X}^{\beta}\left(u^{2}\right)+d_{3} D_{X}^{\beta}\left(v^{2}\right)+d_{4} D_{X}^{\beta} D_{Y}^{\omega} u \\
& +d_{5} D_{X}^{\beta} D_{Y}^{\omega} v+d_{6} D_{X}^{\beta} D_{Y}^{\omega}\left(v^{2}\right)+d_{7} D_{X}^{3 \beta} v+d_{8} D_{X}^{\beta} D_{Y}^{2 \omega} v=0 .
\end{aligned}
$$

It is the time-space fractional coupled gZK equations set. This new set of fractional-order equations will promote the study of fractional-order nonlinear equations and has great significance for the future research.

\section{Solutions of Time-Space Fractional Coupled gZK Equations Set}

In the previous section, the integral order coupled equations set is transformed into the fractional order coupled equations set. To further explore the Rossby solitary waves interaction between two-layer fluids, we solved the time-space fractional coupled gZK equations set by improved $\left(G^{\prime} / G\right)$-expansion method [42,43] in this section.

Firstly, by using the following fractional traveling wave transformations

$$
\begin{gathered}
u(X, Y, T)=\phi_{1}(\xi), \quad v(X, Y, T)=\phi_{2}(\xi) \\
\xi=\frac{k_{1} X^{\beta}}{\Gamma(1+\beta)}+\frac{k_{2} Y^{\omega}}{\Gamma(1+\omega)}-\frac{\sigma T^{\alpha}}{\Gamma(1+\alpha)}
\end{gathered}
$$

where the $k_{1}, k_{2}, \sigma$ are constants, and using chain rule, we have the equations

$$
\begin{aligned}
& D_{T}^{\alpha} u=\rho_{T}^{\prime} \frac{\partial \phi_{1}}{\partial \xi} D_{T}^{\alpha}, \quad D_{T}^{\alpha} v=\rho_{T}^{\prime} \frac{\partial \phi_{2}}{\partial \xi} D_{T}^{\alpha}, \\
& D_{X}^{\beta} u=\rho_{X}^{\prime} \frac{\partial \phi_{1}}{\partial \xi} D_{X}^{\beta}, \quad D_{X}^{\beta} v=\rho_{X}^{\prime} \frac{\partial \phi_{2}}{\partial \xi} D_{X}^{\beta}, \\
& D_{Y}^{\omega} u=\rho_{Y}^{\prime} \frac{\partial \phi_{1}}{\partial \xi} D_{Y}^{\omega}, \quad D_{Y}^{\omega} v=\rho_{Y}^{\prime} \frac{\partial \phi_{2}}{\partial \xi} D_{Y}^{\omega},
\end{aligned}
$$

with $\rho_{T}, \rho_{X}, \rho_{Y}$ are the fractal indexes, without loss of generality we can make $\rho_{T}=\rho_{X}=\rho_{Y}=l$, thereinto $l$ is a constant.

Put the Equation (29) with Equations (30) and (31) into the Equation (28), the time-space fractional coupled equations set can be reduce to the ordinary differential coupled equations set

$$
\begin{aligned}
& -\sigma \phi_{1}^{\prime}+k_{1}\left(c_{1} \phi_{2} \phi_{1}^{\prime}+c_{1} \phi_{1} \phi_{2}^{\prime}+c_{2} \phi_{1} \phi_{1}^{\prime}+c_{3} \phi_{2} \phi_{2}^{\prime}\right)+k_{1} k_{2} l\left[c_{4} \phi_{1}^{\prime \prime}+c_{5} \phi_{2}^{\prime \prime}\right. \\
& \left.+c_{6} \phi_{1} \phi_{1}^{\prime \prime}+c_{6}\left(\phi_{1}^{\prime}\right)^{2}\right]+\left(c_{7} k_{1}^{3} l^{2}+c_{8} k_{1} k_{2}^{2} l^{2}\right) \phi_{1}^{\prime \prime \prime}=0 \\
& -\sigma \phi_{2}^{\prime}+k_{1}\left(d_{1} \phi_{2} \phi_{1}^{\prime}+d_{1} \phi_{1} \phi_{2}^{\prime}+d_{2} \phi_{1} \phi_{1}^{\prime}+d_{3} \phi_{2} \phi_{2}^{\prime}\right)+k_{1} k_{2} l\left[d_{4} \phi_{1}^{\prime \prime}+d_{5} \phi_{2}^{\prime \prime}\right. \\
& \left.+d_{6} \phi_{2} \phi_{2}^{\prime \prime}+d_{6}\left(\phi_{2}^{\prime}\right)^{2}\right]+\left(d_{7} k_{1}^{3} l^{2}+d_{8} k_{1} k_{2}^{2} l^{2}\right) \phi_{2}^{\prime \prime \prime}=0 .
\end{aligned}
$$

Secondly, suppose Equation (32) have the solutions in relation to $\left(G^{\prime} / G\right)$ as follows

$$
\phi_{1}(\xi)=e_{0}+e_{1}\left(\frac{G^{\prime}}{G}\right), \quad \phi_{2}(\xi)=f_{0}+f_{1}\left(\frac{G^{\prime}}{G}\right) .
$$

where $e_{0}, e_{1}, f_{0}, f_{1}$ are computed later, $G=G(\xi)$ satisfies the second ordinary differential equation

$$
G^{\prime \prime}+m G^{\prime}+n G=0 .
$$


thereinto the apostrophe represents derivative with respect to $\xi$ and $m, n$ are parameters.

By substituting Equation (33) with Equation (34) into (32), collecting all terms with the same order of $\left(G^{\prime} / G\right)$, equating each coefficient of the resulting polynomial to zero, we can obtain a set of algebraic equations for $k_{1}, k_{2}, \sigma, l, e_{0}, e_{1}, f_{0}, f_{1}, m$ and $n$.

The last, due to the very complicated coefficients in the equations, the calculation process is extremely complicated, in order to calculate accurate results, take $c_{1}=d_{1}=c_{6}=d_{6}=2, c_{2}=c_{3}=$ $d_{2}=d_{3}=6, c_{4}=c_{5}=c_{7}=c_{8}=d_{4}=d_{5}=d_{7}=d_{8}=1$. Solving the algebraic equations system and subsequently substituting these constants $k_{1}, k_{2}, \sigma, l, e_{0}, e_{1}, f_{0}, f_{1}, m$ and $n$, we get the solutions of the time-space fractional gZK coupled equations set that we want in following as

Case 1:

$$
\begin{gathered}
\sigma=\frac{\left(H_{1} l k_{1}+8 k_{1}\right)\left(I_{1} H_{1}+H_{3}\right)-4\left(2 H_{2} H_{1}+H_{3}\right) k_{1}-I_{1} H_{1} l+H_{4} H_{1} k_{2} l}{4 k_{2}^{2} l}, \quad e_{0}=\frac{I_{1} H_{1}+H_{2}}{4}, \\
e_{1}=e_{1}, \quad f_{0}=-\frac{I_{1} H_{1}+H_{3}}{4 k_{2}^{2} l}, \quad f_{1}=e_{1}, \quad l=l, \quad m=-\frac{H_{1}}{4 k_{2}}, \quad n=0, \\
\left\{\begin{array}{l}
u_{1}=\frac{I_{1} H_{1}+H_{3}}{4} \pm e_{1} \frac{H_{2}}{8 k_{2}}\left[\frac{C_{1} \sinh \left( \pm \frac{H_{2}}{8 k_{2}} \xi\right)+C_{2} \cosh \left( \pm \frac{H_{2}}{8 k_{2}} \xi\right)}{C_{1} \cosh \left( \pm \frac{H_{2}}{8 k_{2}} \xi\right)+C_{2} \sinh \left( \pm \frac{H_{2}}{8 k_{2}} \xi\right)}\right]+\frac{H_{1}}{8 k_{2}}, \\
v_{1}=\frac{I_{1} H_{1}+H_{2}}{4} \pm f_{1} \frac{H_{2}}{8 k_{2}}\left[\frac{C_{1} \sinh \left( \pm \frac{H_{2}}{8 k_{2}} \xi\right)+C_{2} \cosh \left( \pm \frac{H_{2}}{8 k_{2}} \xi\right)}{C_{1} \cosh \left( \pm \frac{H_{2}}{8 k_{2}} \xi\right)+C_{2} \sinh \left( \pm \frac{H_{2}}{8 k_{2}} \xi\right)}\right]+\frac{H_{1}}{8 k_{2}},
\end{array}\right.
\end{gathered}
$$

where $C_{1}, C_{2}$ are arbitrary constants, $H_{1}=2 k_{2} e_{1}-3\left(k_{1}^{2}+k_{2}^{2} l\right), I_{1}=-\left(k_{1}^{2}+k_{2}^{2}\right) l^{2}, H_{2}=2 e_{1} k_{2} l+$ $5 I_{1}, H_{3}=k_{2}^{2} l-4 e_{1} k_{2}, H_{4}=l k_{1} k_{2}-4 e_{1} k_{1}$.

Case 2:

$$
\begin{gathered}
\sigma=\frac{-16 H_{5} k_{1}+I_{2} k_{1} k_{2} l^{2}+I_{3} k_{2} l-4 l^{2} k_{1} k_{2}^{2}, \quad e_{0}=\frac{H_{5}}{k_{2} l}, \quad e_{1}=-\frac{H_{6}}{2 k_{2}},}{f_{0}=-\frac{H_{5}}{k_{2} l}, \quad f_{1}=-\frac{H_{6}}{2 k_{2}}, \quad l=l, \quad m=0, \quad n=\frac{1}{2},} \\
\left\{\begin{array}{l}
u_{2}=\frac{H_{5}}{k_{2} l}-\frac{H_{6} \sqrt{2}}{4 k_{2}}\left[\frac{-C_{1} \sin \left(\frac{\sqrt{2}}{2} \xi\right)+C_{2} \cos \left(\frac{\sqrt{2}}{2} \xi\right)}{C_{1} \cos \left(\frac{\sqrt{2}}{2} \xi\right)+C_{2} \sin \left(\frac{\sqrt{2}}{2} \xi\right)}\right]-\frac{\sqrt{2}}{2}, \\
v_{2}=-\frac{H_{5}}{k_{2} l}-\frac{H_{6} \sqrt{2}}{4 k_{2}}\left[\frac{-C_{1} \sin \left(\frac{\sqrt{2}}{2} \xi\right)+C_{2} \cos \left(\frac{\sqrt{2}}{2} \xi\right)}{C_{1} \cos \left(\frac{\sqrt{2}}{2} \xi\right)+C_{2} \sin \left(\frac{\sqrt{2}}{2} \xi\right)}\right]-\frac{\sqrt{2}}{2},
\end{array}\right.
\end{gathered}
$$

where $C_{1}, C_{2}$ are arbitrary constants, $H_{5}=\frac{k_{1} k_{2}^{2} l-2\left[3\left(k_{1}^{2}+k_{2}^{2}\right) l+4 k_{2}\right] k_{1}}{k_{2}}, I_{2}=-\left(2 k_{1}^{2}+3 k_{2}^{2}\right) k_{1} l^{2}+4 k_{1} k_{2} l$, $I_{3}=4\left(k_{1}^{3}+k_{1} k_{2}^{2}\right) l^{2}, H_{6}=-3\left(k_{1}^{2}+k_{2}^{2}\right) l+4 k_{2}$.

Case 3:

$$
\begin{aligned}
& \sigma=\frac{I_{4} n}{H_{7}^{2}}, \quad e_{0}=-12 \frac{H_{8}}{H_{7}}, \quad e_{1}=\frac{4 k_{2}^{2}}{H_{7}}, \quad f_{0}=36 \frac{H_{8}}{H_{7}}, \\
& f_{1}=0, \quad l=\frac{12 k_{2}}{H_{7}}, \quad m=-2 n+1, \quad n=n,
\end{aligned}
$$

(i) When $4 n^{2}-8 n+2>0$, the hyperbolic solutions as:

$$
\left\{\begin{array}{l}
u_{31}=-12 \frac{H_{8}}{H_{7}}+\frac{4 k_{2}^{2} \sqrt{4 n^{2}-8 n+2}}{2 H_{7}}\left[\frac{C_{1} \sinh \left(\sqrt{4 n^{2}-8 n+2} \xi\right)+C_{2} \cosh \left(\sqrt{4 n^{2}-8 n+2} \xi\right)}{C_{1} \cosh \left(\sqrt{4 n^{2}-8 n+2} \xi\right)+C_{2} \sinh \left(\sqrt{4 n^{2}-8 n+2} \xi\right)}\right]-\frac{1-2 n}{2}, \\
v_{31}=36 \frac{H_{8}}{H_{7}}-\frac{1-2 n}{2}
\end{array}\right.
$$


(ii) When $4 n^{2}-8 n+2>0$, the trigonometric solutions as:

$$
\left\{\begin{array}{l}
u_{32}=-12 \frac{H_{8}}{H_{7}}-\frac{4 k_{2}^{2} \sqrt{4 n^{2}+2}}{2 H_{7}}\left[\frac{-C_{1} \sin \left(\sqrt{4 n^{2}-8 n+2} \xi\right)+C_{2} \cos \left(\sqrt{4 n^{2}-8 n+2} \xi\right)}{C_{1} \cos \left(\sqrt{4 n^{2}-8 n+2} \xi\right)+C_{2} \sin \left(\sqrt{4 n^{2}-8 n+2} \xi\right)}\right]-\frac{1-2 n}{2} \\
v_{32}=36 \frac{H_{8}}{H_{7}}-\frac{1-2 n}{2}
\end{array}\right.
$$

(iii) When $4 n^{2}-8 n+2>0$, the solutions as:

$$
\left\{\begin{array}{l}
u_{33}=\left(\frac{C_{2}}{C_{1}+C_{2} \xi}-\frac{1-2 n}{2}\right), \\
v_{33}=\left(\frac{C_{1}}{C_{2}+C_{1} \xi}-\frac{1-2 n}{2}\right),
\end{array}\right.
$$

where $C_{1}, C_{2}$ are arbitrary constants, $I_{4}=288 k_{1} k_{2}^{2}\left(k_{1}^{2}+k_{2}^{2}\right), H_{7}=9 k_{1}^{2}+7 k_{2}^{2}, H_{8}=2 k_{1}^{2} n+2 k_{2}^{2} n-k_{1}^{2}-k_{2}^{2}$.

The exact solutions obtained by the research show that the fluctuation relationship of each wave not only contains its own wave number and amplitude, but also contains the amplitude of another wave, which explains the main characteristics of nonlinear waves interaction [61]. On the other hand, the interaction between Rossby waves has a great influence on the propagation stability of waves. When both waves are unstable, and they are still unstable after the interaction. When at least one wave is stable, the two waves may be stable or unstable through the interaction, which is related to the values of the coupling term coefficients.

\section{Conclusions}

In this paper, based on the quasi-geostrophic vortex equation set, we obtain the (2+1)-dimensional coupled gZK equations set for the first time, which can describe Rossby solitary waves interactions in two-layer fluids. Next, according to the new model and using the semi-inverse method and the fractional variational principle, a new (2+1)-dimensional time-space fractional coupled gZK equations set is obtained. Then, we solved the (2+1)-dimensional time-space fractional coupled gZK equations set. The coupled gZK equations set is the evolution of a single gZK equation in two-layer fluids, which is of great significance for the study of Rossby waves propagation and interaction. How Rossby solitary waves described by coupled equations set interacts specifically and how the energy changes during the interaction, which are our research aim in the future.

Author Contributions: All authors contributed equally to the writing of this paper. All authors read and approved the final manuscript.

Funding: This work was supported by Nature Science Foundation of Shandong Province of China (No. ZR2018MA017), China Postdoctoral Science Foundation funded project (No. 2017M610436), Open Fund of the Key Laboratory of Meteorological Disaster of Ministry of Education (Nanjing University of Information Science and Technology) (No. KLME201801).

Conflicts of Interest: The authors declare no conflict of interest.

\section{References}

1. Pedlosky, J. Geophysical Fluid Dynamics; Springer-Verlag: New York, NY, USA, 1987.

2. Yang, H.W.; Zhao, Q.F.; Yin, B.S.; Dong, H.H. A new integro-differential equation for Rossby solitary waves with topography effect in deep rotational fluids. Abstr. Appl. Anal. 2013, 2013, 597807. [CrossRef]

3. Guo, M.; Dong, H.Y.; Liu, J.X.; Yang, H.W. The time-fractional mZK equation for gravity solitarywaves and solutions using sech-tanh and radial basic function method. Nonlinear Anal. Model. Control 2018, 24, 1-19. [CrossRef]

4. Caillol, P.; Grimshaw, R.H. Rossby solitary waves in the presence of a critical layer. Stud. Appl. Math. 2007, 118, 313-364. [CrossRef] 
5. Yuan, C.; Grimshaw, R.; Johnson, E.; Chen, X. The propagation of internal solitary waves over variable topography in a horizontally two-dimensional framework. J. Phys. Oceanogr. 2018, 48, 283-300. [CrossRef]

6. Yuan, C.; Grimshaw, R.; Johnson, E.; Wang, Z. Topographic effect on oblique internal wave-wave interactions. J. Fluid Mech. 2018, 856, 36-60. [CrossRef]

7. Preusse, M.; Freistühler, H.; Peeters, F. Seasonal variation of solitary wave properties in Lake Constance. J. Geophys. Res. 2012, 117, C04026. [CrossRef]

8. Ulloa, H.N.; de la Fuente, A.; Niño, Y. An experimental study of the free evolution of rotating, nonlinear internal gravity waves in a two-layer stratified fluid. J. Fluid Mech. 2014, 742, 308-339. [CrossRef]

9. Ulloa, H.N.; de la Fuente, A.; Niño, Y. Degeneration of internal Kelvin waves in a continuous two-layer stratification. J. Fluid Mech. 2015, 777, 68-96. [CrossRef]

10. Hukuda, H. Solitary Rossby waves in a two-layer system. Tellus 1979, 31, 161-169. [CrossRef]

11. Zhao, B.J.; Wang, R.Y.; Sun, W.J.; Yang, H.W. Combined ZK-mZK equation for Rossby solitary waves with complete coriolis force and its conservation laws as well as exact solutions. Adv. Differ. Equ. 2018, 2018, 42. [CrossRef]

12. Yang, H.W.; Chen, X.; Guo, M.; Chen, Y.D. A new ZK-BO equation for three-dimensional algebraic Rossby solitary waves and its solution as well as fission property. Nonlinear Dyn. 2017, 91, 2019-2032. [CrossRef]

13. Tang, X.Y.; Liang, Z.F.; Hao, X.Z. Nonlinear waves of a nonlocal modified KdV equation in the atmospheric and oceanic dynamical system. Commun. Nonlinear Sci. Numer. Simul. 2018, 60, 62-71. [CrossRef]

14. Yin, J.L.; Ding, S.Y.; Tian, L.X.; Fan, X.H.; Deng, X.Y. Existence of exotic waves for the nonlinear dispersive mKdV equation. Appl. Math. Comput. 2014, 229, 499-504. [CrossRef]

15. Lu, C.N.; Fu, C.; Yang, H.W. Time-fractional generalized Boussinesq Equation for Rossby solitary waves with dissipation effect in stratified fluid and conservation laws as well as exact solutions. Appl. Math. Comput. 2018, 327, 104-116. [CrossRef]

16. Yin, X.J.; Yang, L.G.; Liu, Q.S.; Wu, G.R. (2+1)-dimensional ZK-Burgers equation with the generalized beta effect and its exact solitary solution. Comput. Math. Appl. 2019, 77, 302-310. [CrossRef]

17. Yang, H.W.; Guo, M.; He, H.L. Conservation laws of space-time fractional mZK equation for Rossby solitary waves with complete coriolis force. Int. J. Nonlinear Sci. Numer. Simul. 2018. [CrossRef]

18. Zou, L.; Yu, Z.B.; Wang, X.B. Dynamics of the breather waves, rogue waves and solitary waves in an extend Kadomtsev-Petviashvili equation. Appl. Math. Lett. 2018, 83, 73-79. [CrossRef]

19. Bracco, A.; Pedlosky, J. Votex generation by topopraphy in locally unstable baroclinic flows. J. Phys. Oceanogr. 2003, 33, 207-219. [CrossRef]

20. Atangana, A.; Secer, A. The time-fractional coupled-Korteweg-de-Vries equations. Abstr. Appl. Anal. 2013, 2013, 947986. [CrossRef]

21. Eslami, M. Exact traveling wave solutions to the fractional coupled nonlinear Schrodiger equations. Appl. Math. Comput. 2016, 285, 141-148.

22. Lou, S.Y.; Tong, B.; Hu, H.C.; Tang, X.Y. Coupled KdV equations derived from two-layer fluids. J. Phys. Math. Gen. 2005, 38, 1-15. [CrossRef]

23. Farwig, R.; Qian, C. Asymptotic behavior for the quasi-geostrophic equations with fractional dissipation in $R^{2}$. J. Differ. Equ. 2018. [CrossRef]

24. Fu, C.; Lu, C.N.; Yang, H.W. Time-space fractional $(2+1)$-dimensional nonlinear Schrodinger equation for envelope gravity waves in baroclinic atmosphere and conservation laws as well as exact solutions. Adv. Differ. Equ. 2018, 2018, 56. [CrossRef]

25. Yang, H.W.; Yin, B.S.; Shi, Y.L; Wang, Q.B. Forced ILW-Burgers equation as a model for Rossby solitary waves generated by topography in finite depth fluids. J. Appl. Math. 2012, 2012, 491343. [CrossRef]

26. Yang, H.W.; Xu, Z.H.; Yang, D.Z.; Feng, X.R.; Yin, B.S.; Dong, H.H. ZK-Burgers equation for three-dimensional Rossby solitary waves and its solutions as well as chirp effect. Adv. Differ. Equ. 2016, 2016, 167. [CrossRef]

27. Yang, X.J.; Machado, J.A.T.; Baleanu, D. Exact traveling-wave solution for local fractional boussinesq equation in fractal domain. Fractals 2017, 25, 1740006. [CrossRef]

28. Guo, M.; Fu, C.; Zhang, Y.; Liu, J.X.; Yang, H.W. Study of Ion-Acoustic solitary waves in a magnetized plasma using the three-dimensional time-space fractional Schamel-KdV equation. Complexity 2018, 2018, 6852548. [CrossRef] 
29. Zhang, L.F.; He, Z.H.; Conti, C.; Wang, Z.T.; Hu, Y.H.; Lei, D.J.; Li, Y.; Fan, D.Y. Modulational instability in fractional nonlinear Schrodinger equation. Commum. Nonlinear Sci. Numer Simul. 2017, 48, 531-540. [CrossRef]

30. Yang, H.W.; Sun, J.C.; Fu, C. Time-fractional Benjamin-Ono equation for algebraic gravity solitary waves in baroclinic atmosphere and exact multi-soliton solution as well as interaction. Commun. Nonlinear Sci. Numer. Simul. 2019, 71, 187-201. [CrossRef]

31. I-Wakil, S.A.E.; Abulwafa, E.M.; I-Shewy, E.K.E.; Mahmoud, A.A. Ion-acoustic waves in unmagnetized collisionless weakly relativistic plasma of warm-ion and isothermal-electron using time-fractional $\mathrm{KdV}$ equation. Sci. Verse Sci. Direct. 2012, 49, 1721-1727.

32. Ma, W.X.; Yong, X.L.; Zhang, H.Q. Diversity of interaction solutions to the (2+1)-dimensional Ito equation. Comput. Math. Appl. 2018, 75, 289-295. [CrossRef]

33. Wang, X.H.; Wang, Z.; Huang, X.; Li, Y.X. Dynamic analysis of a fractional-order delayed SIR model with saturated incidence and treatment functions. Int. J. Bifurc. Chaos 2018, 28, 1850180.

34. Yang, X.J.; Gao, F.; Srivastava, M.H. A new computational approach for solving nonlinear local fractional PDEs. J. Comput. Appl. Math. 2018, 339, 285-296. [CrossRef]

35. Ma, W.X.; Zhou, Y. Lump solutions to nonlinear partial differential equations via hirota bilinear forms. J. Differ. Equ. 2018, 264, 2639-2659. [CrossRef]

36. Zhao, H.Q.; Ma, W.X. Mixed lump-kink solutions to the KP equation. Comput. Math. Appl. 2017, 74, 1399-1405. [CrossRef]

37. Guner, O.; Aksoy, E.; Bekir, A.; Cevikel, A.C. Different methods for (3+1)-dimensional space-time fractional modified KdV-Zakharov-Kuznetsov equation. Comput. Math. Appl. 2016, 71, 1259-1269. [CrossRef]

38. Guo, M.; Zhang, Y.; Wang, M.; Chen, Y.D.; Yang, H.W. A new ZK-ILW equation for algebraic gravity solitary waves in finite depth stratified atmosphere and the research of squall lines formation mechanism. Comput. Math. Appl. 2018, 75, 3589-3603. [CrossRef]

39. Tao, M.S.; Dong, H.H. Algebro-Geometric solutions for a discrete integrable equation. Discret. Dyn. Nat. Soc. 2017, 2017, 5258375. [CrossRef]

40. Zou, Y.M.; Cui, Y.J. Existence results for a functional boundary value problem of fractional differential equations. Adv. Differ. Equ. 2013, 1, 233. [CrossRef]

41. Bai, Z.B.; Sun, W.C. Existence and multiplicity of positive solutions for singular fractional boundary value problems. Comput. Math. Appl. 2012, 63, 1369-1381. [CrossRef]

42. Sahoo, S.; Ray, S.S. Solitary wave solutions for time fractional third order modified KdV equation using two raliable techniques $\left(G^{\prime} / G\right)$-expansion method and improved $\left(G^{\prime} / G\right)$-expansion method. Phys. A 2016, 448, 265-282. [CrossRef]

43. Islam, M.N.; Akbar, M.A. New exact wave solutions to the space-time fractional coupled Burgers equationa and the space-time fractional foam drainage equation. Cog. Phys. 2018, 5, 1422957.

44. Sahoo, S.; Ray, S.S. Improved fractional sun-equation method for (3+1)-dimensional generalized fractional KdV-Zakharov-Kuznetsov equations. Comput. Math. Appl. 2015, 70, 158-166. [CrossRef]

45. He, J.H.; Wu, G.C.; Austin, F. The variational iteration method which should be followed. Nonlinear Sci. Lett. A Math. Phys. Mech. 2010, 1, 1-30.

46. Shallal, M.A.; Jabbar, H.N.; Ali, K.K. Analytic solution for the space-time fractional Klein-Gordon and coupled conformable Boussinesq equations. Results Phys. 2018, 8, 372-378. [CrossRef]

47. Khater, M.A.; Seadawy, A.R.; Lu, D. Elliptic and solitary wave solutions for Bogoyavlenskii equations system, coupled Boiti-Leon-Pempinelli equations system and time-fractional Cahn-Allen equation. Results Phys. 2017, 7, 2325-2333. [CrossRef]

48. Zeidan, D. Assessment of mixture two-phase flow equations for volcanic flows using Godunov-type methods. Appl. Math. Comput. 2016, 272, 707-719. [CrossRef]

49. Tao, M.S.; Zhang, N.; Gao, D.Z.; Yang, H.W. Symmetry analysis for three-dimensional dissipation Rossby waves. Adv. Differ. Equ. 2018, 2018, 300. [CrossRef]

50. Ma, W.X. Conservation laws by symmetries and adjoint symmetries. Discret. Conti. Dyn. Syst. Ser. S 2018, 11, 707-721.

51. Shi, Y.L.; Yin, B.S.; Yang, H.W.; Yang, D.Z.; Xu, Z.H. Dissipative nonlinear Schrodinger equation ror envelope solitary Rossby waves with dissipation effect in stratified fluids and its solution. Abstr. Appl. Anal. 2014, 2014, 643652. [CrossRef] 
52. I-Wakil, S.A.E.; Abulwafa, E.M. Formulation and solution of space-time fractional Boussinesq equation. Nonlinear Dyn. 2015, 80, 167-175. [CrossRef]

53. Agrawal, O.P. Formulation of Euler-Lagrange equation for fractional variational problems. J. Math. Anal. Appl. 2002, 272, 368-379. [CrossRef]

54. I-Wakil, S.A.E.; Abulwafa, E.M.; Zahran, M.A.; Mahmoud, A.A. Time-fractional KdV equational: formulation and solution using variational methods. Nonlinear Dyn. 2011, 65, 55-63. [CrossRef]

55. He, J.H. A tutorial and heuristic review on Lagrange multiplier for optimal problems. Nonlinear Sci. Lett. A 2017, 8, 121-148. [CrossRef]

56. He, J.H. Fractal calculus and its geometrical explanation. Results Phys. 2018, 10, 272-276.

57. Baleanu, D.; Muslih, S.I. Lagrangian formulationof classical fields within Riemann-Liouville fractional derivatives. Phys. Scr. 2005, 72, 119-123. [CrossRef]

58. Agrawal, O.P. A general formulation and solution scheme for fractional optimal control problems. Nonlinear Dyn. 2004, 38, 323-337. [CrossRef]

59. Agrawal, O.P. Fractional variational calculus and thetransversality conditions. J. Phys. A Math. Gen. 2006, 39, 10375-10348. [CrossRef]

60. Agrawal, O.P. Fractional variational calculus in terms of Riesz fractional derivatives. J. Phys. A Math. Theor. 2007, 40, 6287-6303. [CrossRef]

61. Zeidan, D.; Sekhar, T.R.On the wave interactions in the drift-flux equations of two-phase flows. Appl. Math. Comput. 2018, 327, 117-131. [CrossRef]

(C) 2019 by the authors. Licensee MDPI, Basel, Switzerland. This article is an open access article distributed under the terms and conditions of the Creative Commons Attribution (CC BY) license (http://creativecommons.org/licenses/by/4.0/). 